\title{
Studies of Beam Induced Radiation Backgrounds for the Cosmic Ray Veto Detector Operations at the Mu2e Experiment
}

\author{
Yuri Oksuzian*广 \\ University of Virginia \\ E-mail: oksuzianefnal.gov
}

\begin{abstract}
The Mu2e experiment will search for a neutrinoless muon-to-electron conversion process with almost four orders of magnitude of sensitivity improvement relative to the current best limit. One important background is caused by cosmic-ray muons and their secondaries faking the conversion electron signature. In order to reach the designed sensitivity, Mu2e needs to identify cosmic-ray muons with an efficiency of $>99.9 \%$. The Cosmic Ray Veto (CRV) detector consists of four layers of plastic scintillator which surrounds the Mu2e detector, with an area of approximately 300 square meters. The CRV must operate in the presence of large neutron and gamma fluxes produced from beam interactions. This radiation can damage the detector components and generate significant background rates in the CRV. We estimate the background rates and the total dead-time produced by these backgrounds using a simulation which includes a sophisticated model of the Mu2e apparatus and the CRV response.
\end{abstract}

38th International Conference on High Energy Physics

3-10 August 2016

Chicago, USA

\footnotetext{
*Speaker.

${ }^{\dagger}$ On behalf of the Mu2e Collaboration.
} 
The Mu2e experiment will search for the conversion of a muon into an electron in the presence of a nucleus [1] at a sensitivity of about four orders of magnitude beyond the best current limits. The observation of this process would be an unambiguous sign of physics beyond the standard model.

At Mu2e [Fig. 1], a bunched proton beam delivers a low-momentum muons to the Aluminum stopping target every 1695 ns. Stopped muons interact with Aluminum nuclei, and the signal would produce a mono-energetic electron with energy of $105 \mathrm{MeV}$. In order to reach the design single-event sensitivity of $2.9 \times 10^{-17}$, about $10^{18}$ muons must be stopped over three years.

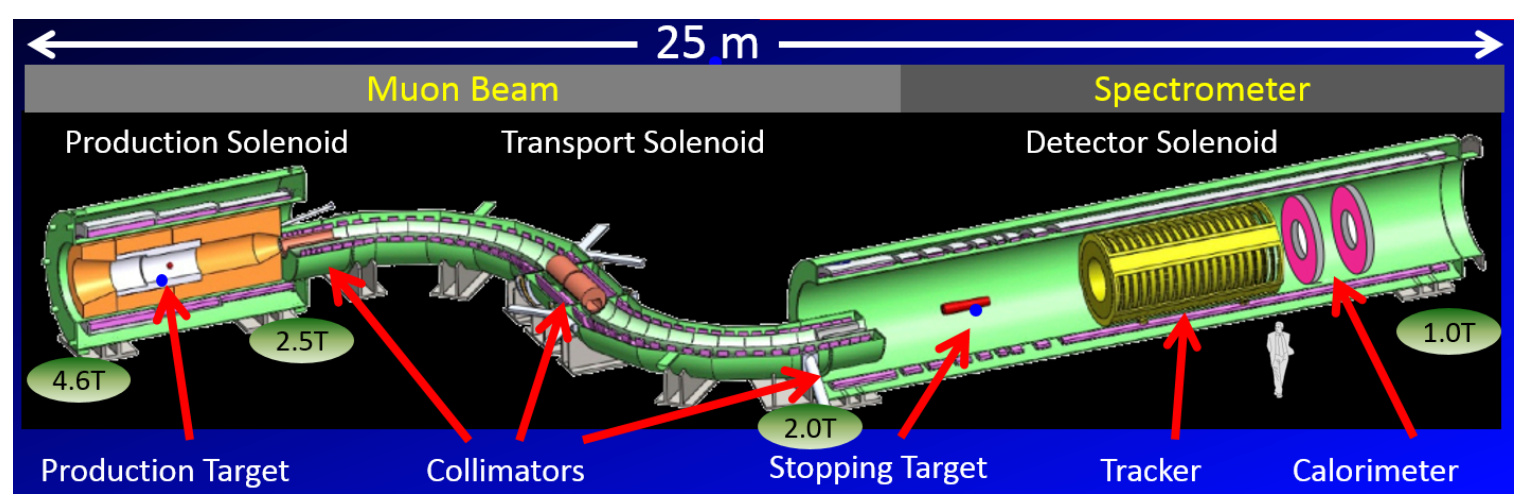

Figure 1: The Mu2e experimental setup. The pulsed $8 \mathrm{GeV}$ proton beam strikes the production target in the production solenoid. Produced muons are transported through the transport solenoid to the stopping target. Electrons produced from stopped muons are measured by the tracker and the calorimeter. The Cosmic Ray Veto (CRV) surrounds the detector solenoid region, and is shown in Fig. 4

Keeping the background expectation to less than one event in this high-intensity experiment is a challenge. One type of background is prompt, induced by $\pi^{-}$radiative capture on the stopping target. Taking advantage of the muon lifetime and pulsed beam structure are used to control prompt backgrounds. After a beam pulse there is a delay of $675 \mathrm{~ns}$ before the time window begins and reconstructed electrons are considered signal candidates. Another type of background is induced by cosmic ray muons which produce about one conversion-like background per day. The CRV, a 4-layers polystyrene scintillator detector, covers the detector solenoid and detects cosmic muons by requiring signal hits in at least 3 layers, localized in time and space. After a cosmic ray muon detection, time interval of $125 \mathrm{~ns}$ is removed from the search window in the offline analyses.

CRV muon detection efficiency of $>99.9 \%$ needs to be maintained in the beam induced high radiation environment. The major source of radiation impacting the CRV operations is neutrons and their products, gammas:

(a) Neutron and gamma interactions with the CRV scintillator produce readout noise, which can fake cosmic ray muons and consequently increase the dead-time for the experiment.

(b) Background noise degrades the CRV timing resolution, which plays a crucial part in coincidence formation between CRV layers.

(c) High energy neutrons damage the CRV readout components.

In order to estimate mentioned impacts, Geant4 [2] based simulation was used which includes a model of the Mu2e apparatus [Fig. 3] and the CRV response. 


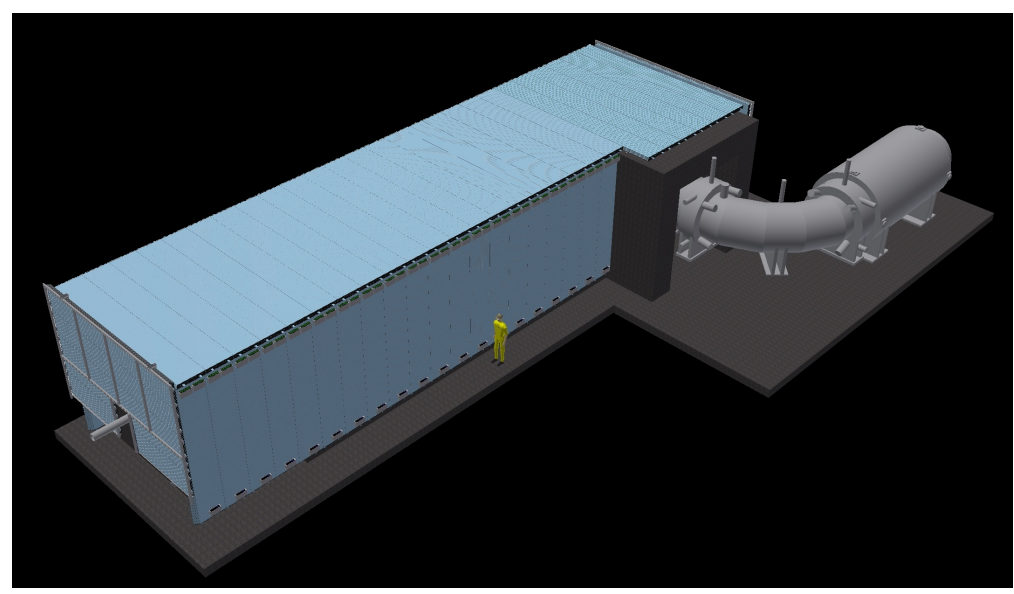

Figure 2: The CRV, covering the detector solenoid region and is composed of 4 layers of extruded polystyrene scintillator counters and embedded wavelength shifting fibers, read out by silicon Pphotomultipliers (SiPM).

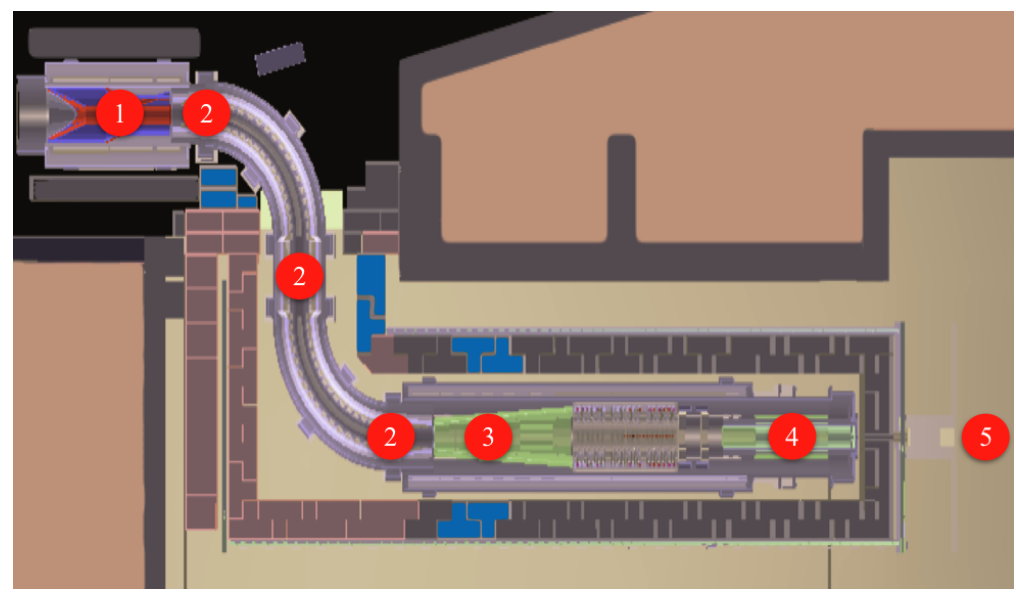

Figure 3: Mu2e geometry in Geant4 simulation framework. (1) - production solenoid, (2) - collimators, (3) - stopping target, (4) - MBS, (5) - stopping target monitor shielding, brown - boron concrete, blue - barite concrete, grey - regular concrete.

The simulation reveal multiple sources of radiation backgrounds that produce challenges to the CRV operations:

(1) Largest source of neutrons at Mu2e originate from the primary proton beam interaction with the tangstum target in the production solenoid. Neutrons get thermalized by the time Mu2e opens up the signal window. Thermal neutrons are captured within several ms and produce gammas with energies of up to $10 \mathrm{MeV}$. Gammas of these energies can deposit a significant energy in the scintillator to fake a hit from cosmic a muon. To reduce the gamma spectrum from neutron captures, upstream concrete shielding is loaded with $0.7 \%$ boron carbide. Boron yields high neutron capture cross-section with a low (478 KeV) energy gamma emission.

(2) Muons stopped on the $\mathrm{Al}$ target are captured with a branching ratio of $61 \%$, producing fast ( $>1$ $\mathrm{MeV}$ ) neutrons. These neutrons are produced inside the signal window, and can directly produce 
hits in the CRV. High-Z barite enriched concrete surronds the stopping target region to reduce the neutron energies.

(3) Only half of all muons reaching the stopping target stop on the Aluminum foils, and the remaining fraction of "unused" muons is dumped into the muon beam stop (MBS). MBS consists of low-Z polyethylene barrel. Muons stopped on a low-Z material decay to electrons without producing harmful neutrons. A small fraction of gammas produced by electron brems is shielded with concrete.

(4) The stopping target monitor, located outside of the shielding and designed to measure the rate of stopped muons, requires a field of view to the stopping target. Muons escaping the shielding decay producing high energy Michel electrons. Electrons of these energies can penetrate through 3 layers of the CRV, mimicking a cosmic muon. A thin $(1 \mathrm{~cm})$ layer of polyethylene absorber in the end region of the MBS stops a large fraction of these low-momentum muons, and the remaining fraction is shielded by the stopping target monitor shielding.

Based on the results of the simulations, the total dead-time to the Mu2e experiment generated by the beam induced radiation in the CRV is estimated to be $<10 \%$. The radiation damage to the electronics readout components and mainly to SiPMs is estimated from the total expected neutron flux normalized to the $1 \mathrm{MeV}$ neutron induced displacement equivalent [3]. The average neutron rates at the $\mathrm{CRV}$ readout is $5 \mathrm{E} 8\left[1 \mathrm{MeVeq}\right.$ neutron] $/ \mathrm{cm}^{2}$, which is lower than the required rate of $1 \mathrm{E} 10[1 \mathrm{MeVeq}$ neutron $] / \mathrm{cm}^{2}$.

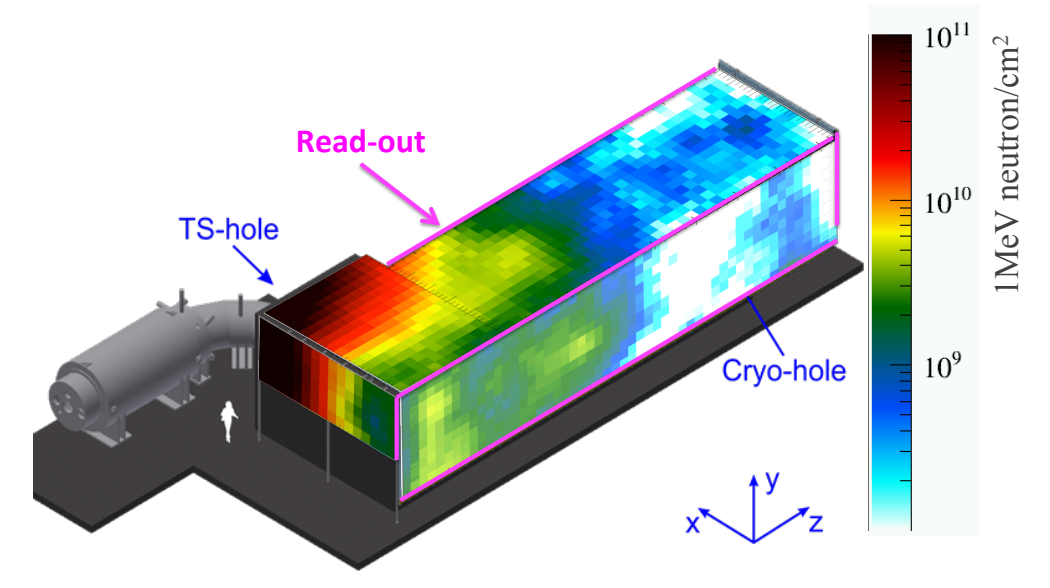

Figure 4: Radiation damage rates to the CRV readout components induced by neutrons. Total damage rates SiPM can withstand are $1 \mathrm{E} 10[1 \mathrm{MeVeq}$ neutron $] / \mathrm{cm}^{2}$

\section{References}

[1] L. Bartoszek, et al., Mu2e Technical Design Report, arXiv:1501.05241.

[2] S. Agostinelli, et al., Geant4 - a simulation toolkit, Nucl. Instrum. Methods A, 506 (2003), p. 250

[3] A. Vasilescu and G. Lindstroem, Displacement Damage in Silicon, http://rd50.web.cern.ch/rd50/NIEL/default.html. 